\title{
A Stochastic Model of a Transport Hub and Multi-phase Queueing Systems
}

\author{
Anna A. Lempert, Alexander L. Kazakov, Maxim L. Zharkov
}

Matrosov Institute for system dynamics and control theory of Siberian Branch of the Russian Academy of Sciences Lermontov str., 134

Irkutsk, Russia

e-mail: lempert@icc.ru

\begin{abstract}
The paper is devoted to designing of a stochastic model of a transport hub. As a mathematical apparatus, we use multiphase queuing systems with an incoming BMAP-flow. The model is identified on the basis of statistical data and field observation and tested on two passenger hubs in Moscow. The results of simulation show that the model makes it possible to give an overall assessment of the effectiveness of the transport hub.
\end{abstract}

Keywords: Transport hub, mathematical modelling, multiphase queuing system, BMAP-flow, simulation.

\section{Introduction}

High intensity and density of traffic flows are typical features of large, rapidly developing cities. This leads to congestion of the road network, the appearance of "traffic jams" and a decrease in the speed of movement of ground transport within the city. An effective way to solve these problems is to organize a system of highspeed urban and suburban multimodal transport [1]. For this, we need for special passenger terminals, which ensure a quick and convenient transfer to another transport type. A transport hub (also transport interchange) is a place where passengers and cargo are exchanged between vehicles or between transport modes [2]. As mentioned in [3, 4, 4] the integration of land-use planning and transport planning is as the keystone of a sustainable transport planning.

Note that transport hubs have a complex structure and are subject to the influence of a large number of factors, both deterministic and stochastic. In such cases, mathematical modeling and simulation allows for a better understanding of the ongoing processes and increase in the efficiency of the functioning of such systems.

In the paper we suggest a stochastic mathematical model of transport hubs base on multi-phase queueing systems. The model was identified on the basis of statistical data and field observation. The computational experiment is carried out, the results are discussed.

\section{General Approach}

Here we suggest an approach to the construction of mathematical models of micrologistical transport systems (micro-TS), in particular, transport hubs. It consists of following stages. They are a description of an incoming flow structure, a decomposition of microTS on the levels of service and grouping them into the phases, and a description of an outgoing flow structure.

Incoming flow is determined by two parameters: the time intervals between successive requests and the size of the incoming group. Each of them can be fixed, or given by some random variable.

By studying various micro-TS, we defined a basic service level structure, which consists of three independent, sequentially arranged subsystems. Each of them is a separated queuing system (QS).

The structure of the outgoing flow is given by a random process, the parameters of which, as a rule, cannot be obtained analytically and are calculated approximately by computational experiment.

For the considered micro-TS within the bounds of this approach, four levels are distinguished (see fig. 1). The first level is an incoming flow; the second one is a level of sorting in cargo micro-TS or a level of reception of passengers. Next level is the request processing or passenger services. The last one is an outgoing flow level. 


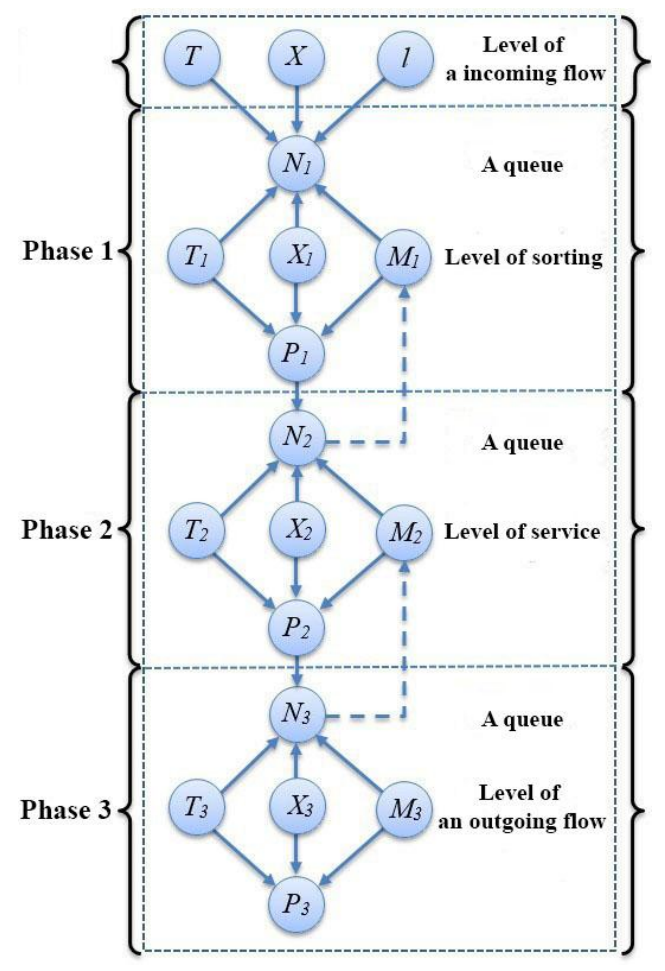

Fig. 1. The structural diagram of mathematical model of micro-TS operation.

Each level can be described by the set of random variables (see fig. 1). Let $T, T_{1}, T_{2}, T_{3}$ be the inter-arrival time of requests groups and $X, X_{1}, X_{2}, X_{3}$ be a size of the arrived group at the respective level. $M_{k}(t)$ be the number of operating channels at the corresponding level at time $t, k=\overline{1,3}$.

The phase is formed by adding the corresponding queue to each level of service. $N_{k}(t)$ be the length of the queue at the phase $k$ at time $t, P_{k}(t)$ be an outgoing flow, $k=\overline{1,3}$.

Between the phases there are feedbacks. This means that the channels of the previous phase are blocked, if in the current phase there is no possibility to accept a requests group.

In terms of queuing theory $[1,6,7]$, we have the system

$$
B M A P / G^{X_{1}} / m_{1} / n_{1} \rightarrow / G^{X_{2}} / m_{2} / n_{2} \rightarrow / G^{X_{3}} / m_{3} / n_{3},
$$

where $X_{i}$ is the maximum size of the served group or the distribution of the size of the served group in phase $i, G$ is arbitrary service time, $m_{i}$ is the number of the channels and $n_{i}$ is the length of the queue at the phase $k$.

\section{Transport hub}

We study two transport hubs located in Moscow. They are Vladykino and Kutuzovo hubs. These systems are chosen as typical hubs, formed on the basis of "concourse". In both hubs, there is a terminal that allows passengers to move from the Moscow central circle platform to the metro station on the principle of "dry feet", that is, without going outside.

There are four types of transport in Vladykino and Kutuzovo: personal vehicles (P) [8], ground public transport (B), metro (M), Moscow central circle (MCC). Besides, there is a flow of passengers from the adjacent territory (pedestrians). These hubs are the most popular on the MCC, the average daily flows are 18.3 and 14.9 thousand people respectively.

Now we determine the main flows of passengers passing through the hubs (see fig. 2).

I. From MCC, B and $\mathrm{P}$ to $\mathrm{M}$.

II. From M, B and P to MCC.

III. MCC and $\mathrm{M}$ to $\mathrm{B}$ and $\mathrm{P}$ (if exists).

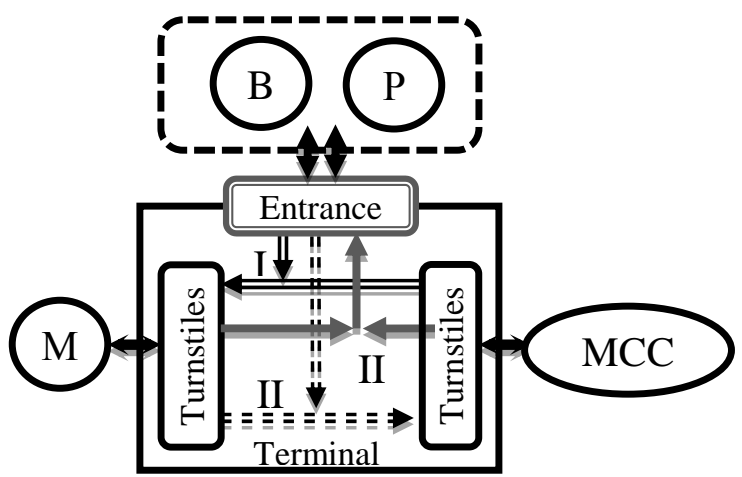

Fig. 2. Scheme of passenger flows.

One can see that a typical transport hub includes several independent systems of various modes of transport. Their functioning is connected with the level of correspondence of passenger flows. This follows from the fact that the outgoing flow from one node of the system is the incoming flow for the other (see Fig. 2). Considering these flows, we find that the movement of passengers can be described by a general algorithm (Fig. 3).

This scheme and the algorithm for the movement of passengers can be used to describe the work of transport hubs with a different structure.

Consider the independent elements of the transport hub (MCC station, metro, bus stop, parking) separately. Passenger traffic has two directions: from the terminal to a vehicle (bus, subway car or other) and from a vehicle to the terminal. In the first case, the vehicle can be considered a service channel, and the platform as a queue. In the second case, the vehicle is the source of passenger traffic, the platform is a queue, and turnstiles at the exit from the platform are servicing channels. The platform has the finite capacity. We assume that passenger flows in both directions are independent. 
Then the model of such elements of a transport hub is a combination of two independent queuing systems with finite queues. Taking into account the traffic flow algorithm, the model of a transport hub is a set of interconnected and sequentially located queuing systems, i.e. multiphase system with finite queues.

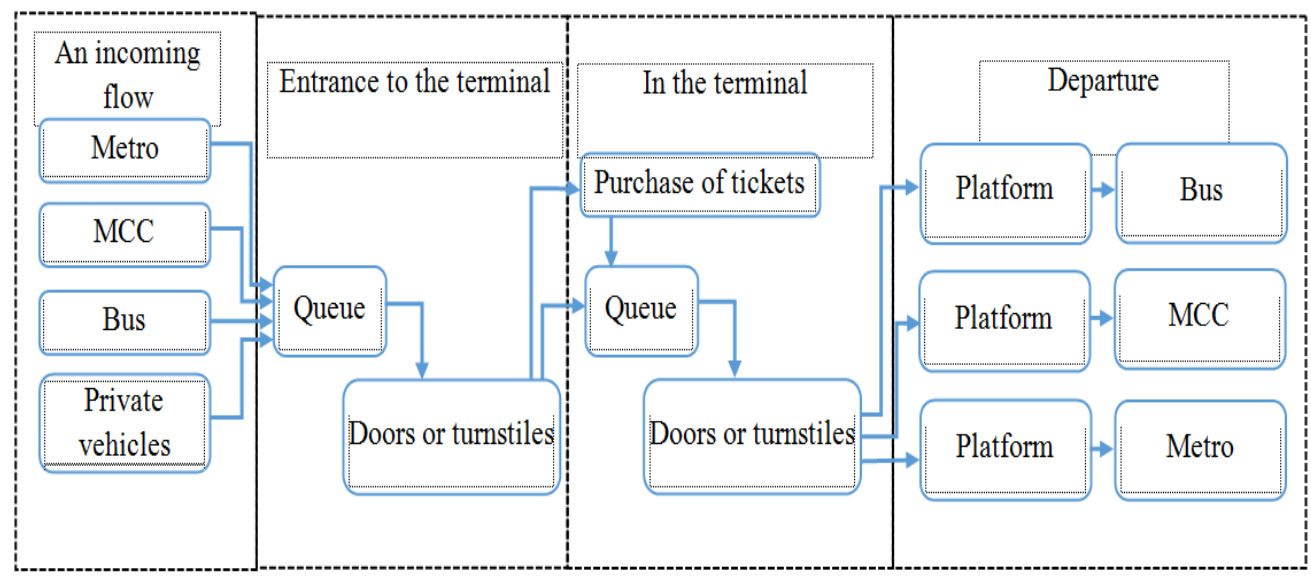

Fig. 3. The algorithm for the movement of passengers through the terminal of transfer hub.

Thus, in constructing a generalized model of the transport hub operation, we will rely on the following properties:

- $\quad$ each node of a transport hub can be represented as a set of two independent queuing systems with finite queues;

- the outgoing flow from a node of a transport hub is incoming one for another;

- there are several passenger flows and the movement of passengers can be described by a general algorithm (Fig. 3).

Tables 1 and 2 presents the main characteristics of the considered transport hubs.

Table 1. The main characteristics of Vladykino hub.

\begin{tabular}{|c|c|c|c|}
\hline & \multicolumn{3}{|c|}{ Vladykino hub } \\
\hline & $\begin{array}{c}\text { Turnstiles } \\
\text { (enter/exit) }\end{array}$ & $\begin{array}{c}\text { Area } \\
\text { (sq.m.) }\end{array}$ & Capacity \\
\hline $\begin{array}{c}\text { Metro } \\
\text { platform }\end{array}$ & $8 / 8$ & 1560 & 3000 \\
\hline $\begin{array}{c}\text { MCC } \\
\text { platform }\end{array}$ & $5 / 5$ & 2520 & 5000 \\
\hline Bus stop & - & 500 & 1000 \\
\hline Terminal & $6 / 6$ & 4250 & 9500 \\
\hline
\end{tabular}

At the entrance to the MCC platforms and in the metro there are turnstiles. The intensity of service is about 20 people per minute. The average throughput of doors in terminals is assumed to be 20 people per minute. We suppose the service time to be a random variable with an exponential distribution. For arriving passengers, we allocate $30 \%$ of the capacity of the
MCC platforms, metro and bus stops, the rest places are for passengers leaving the transport hub. This separation is logical since arrived passengers usually do not stay on the platforms but immediately go to the terminal.

Table 2. The main characteristics of Kutuzovo hub.

\begin{tabular}{|c|c|c|c|}
\hline & \multicolumn{3}{|c|}{ Kutuzovo hub } \\
\hline & $\begin{array}{c}\text { Turnstiles } \\
\text { (enter/exit) }\end{array}$ & $\begin{array}{c}\text { Area } \\
\text { (sq.m.) }\end{array}$ & Capacity \\
\hline $\begin{array}{c}\text { Metro } \\
\text { platform }\end{array}$ & $8 / 8$ & 1560 & 3000 \\
\hline $\begin{array}{c}\text { MCC } \\
\text { platform }\end{array}$ & $4 / 4$ & 1260 & 2500 \\
\hline Bus stop & - & 500 & 1000 \\
\hline Terminal & $6 / 6$ & 1000 & 2000 \\
\hline
\end{tabular}

According to proposed general approach we design the model of the transport hubs in the form of threephase queueing system. Each phase represents the level of service in the transport hub.

Phase 1 is the passage from a vehicle to the terminal. It is simulated by a multichannel QS with a common queue. The distribution of channels over the served flows is shown in Tables 3 and 4.

Phase 2 is the passage from the terminal to the platform for boarding a vehicle (MCC, metro or bus). The characteristics are shown in Tables 3 and 4.

In phases 1 and 2 , the service intensity for each channel is 20 people per minute. 
Table 3. The characteristics of QS on phase 1 and phase 2 for Vladykino hub

\begin{tabular}{|c|c|c|c|}
\hline \multirow{2}{*}{$\begin{array}{c}\text { Passenger } \\
\text { flow }\end{array}$} & \multicolumn{3}{|c|}{ Vladykino hub } \\
\cline { 2 - 2 } & $\begin{array}{c}\text { Number of } \\
\text { channels }\end{array}$ & \multicolumn{2}{|c|}{ the length of the queue } \\
\cline { 2 - 2 } I & 8 & phase 1 & phase 2 \\
\hline II & 5 & 1500 & 9500 \\
\hline III & 6 & & \\
\hline
\end{tabular}

Table 4. The characteristics of QS on phase 1 and phase 2 for Kutuzovo hub.

\begin{tabular}{|c|c|c|c|}
\hline \multirow{2}{*}{$\begin{array}{c}\text { Passenger } \\
\text { flow }\end{array}$} & \multicolumn{3}{|c|}{ Kutuzovo hub } \\
\cline { 2 - 3 } & $\begin{array}{c}\text { Number of } \\
\text { channels }\end{array}$ & \multicolumn{2}{|c|}{ The length of the queue } \\
\hline I & 8 & \multirow{2}{*}{1500} & \multirow{2}{*}{2000} \\
\hline II & 4 & & \\
\hline III & 6 & & \\
\hline
\end{tabular}

Phase 3 is boarding and departure from the system. It consists of three separate queuing systems that have one channel and individual queue. Here, each queueing system serves only one passenger flow of the appropriate type. QS I is a metro platform, QS II is a MCC platform, QS III is a bus stop. The characteristics of Phase 3 are shown in Tables 5 and 6.

Table 5. The characteristics of QS on phase 3 for Vladykino hub.

\begin{tabular}{|c|c|c|c|}
\hline & \multicolumn{3}{|c|}{ Vladykino hub } \\
\hline Phase 3 & $\bar{t}$ & $\begin{array}{c}\text { the length of the } \\
\text { queue }\end{array}$ & $V$ \\
\hline QS I & 2,5 & 2500 & $N(200,30)$ \\
\hline QS II & 6 & 4500 & $N(200,30)$ \\
\hline QS III & 1,25 & 500 & $N(15,4)$ \\
\hline
\end{tabular}

Table 6. The characteristics of QS on phase 3 for Kutuzovo hub.

\begin{tabular}{|c|c|c|c|}
\hline & \multicolumn{3}{|c|}{ Kutuzovo hub } \\
\hline Phase 3 & $\bar{t}$ & $\begin{array}{c}\text { the length of } \\
\text { the queue }\end{array}$ & $V$ \\
\hline QS I & 2,5 & 2500 & $N(200,30)$ \\
\hline QS II & 6 & 2000 & $N(200,30)$ \\
\hline QS III & 0,95 & 500 & $N(15,4)$ \\
\hline
\end{tabular}

Here $\bar{t}$ is average service time (min), $V$ is the size of a serviced group of passengers, and $V$ is a random variable. This assumption is based on two facts. First, during the rush hour it is possible the arrival of an overfull transport, then not all the passengers can board and they have to wait for the next one. Secondly, public transport has two directions of traffic, therefore not all passengers tend to get on the first arrived transport.

\section{Incoming flow}

As shown in the previous section, the transport hub receives five incoming passenger flows. To describe the general incoming passenger traffic, we suggest using the model of $B M A P$-flow.

$B M A P$-flow (or process) is a generalization of the group Poisson process, allowing the change in the intensity of the arrival of request groups, but keeping the basic Markov structure [9,10].

We have a Markov chain $v_{t}$ with continuous time and state space $\{0,1, \ldots, W\}$. The residence time in each state is exponentially distributed with parameter $\lambda_{i}, i=\overline{0, W}$. With probability $p_{(i, r)}^{(k)}, 0 \leq k \leq m$ the process can go to state $r$. This generates a group of random size $k$. The normalization condition is satisfied

$$
\sum_{r=1, r \neq i}^{m} p_{(i, r)}^{(0)}+\sum_{k=1}^{\infty} \sum_{r=1}^{m} p_{(i, r)}^{(k)}=1 .
$$

The transitions intensities are written in matrix form

$$
\begin{gathered}
\left(D_{0}\right)_{i i}=-\lambda_{i}, \quad 0 \leq i \leq W ; \\
\left(D_{0}\right)_{i r}=\lambda_{i} p_{(i, r)}^{(0)}, \quad i, r=\overline{0, W} ; \\
\left(D_{k}\right)_{i r}=\lambda_{i} p_{(i, r)}^{(k)}, \quad i, r=\overline{0, W} ; k \geq 1 .
\end{gathered}
$$

Based on the processing of statistical data, we obtained the following characteristics of the incoming passenger flows (tables 7 and 8).

Table 7. Incoming flow to Vladykino hub.

\begin{tabular}{|l|l|l|l|}
\hline & Intensity & $\begin{array}{l}\text { Probability } \\
\text { of arrival }\end{array}$ & $\begin{array}{l}\text { Distribution } \\
\text { law }\end{array}$ \\
\hline Metro & 0.8 & 0.142 & $N(170,46)$ \\
\hline MCC & 0.34 & 0.06 & $N(120,40)$ \\
\hline Buses & 0.78 & 0.138 & $N(27,8,7)$ \\
\hline Cars & 1.41 & 0.25 & Geom $(0.75)$ \\
\hline Pedestrians & 2.31 & 0.41 & $N(7,2)$ \\
\hline
\end{tabular}

Table 8. Incoming flow to Kutuzovo hub.

\begin{tabular}{|l|l|l|l|}
\hline & Intensity & $\begin{array}{l}\text { Probability } \\
\text { of arrival }\end{array}$ & $\begin{array}{l}\text { Distribution } \\
\text { law }\end{array}$ \\
\hline Metro & 0.8 & 0.268 & $N(170,46)$ \\
\hline MCC & 0.34 & 0.114 & $N(120,40)$ \\
\hline Buses & 1.15 & 0.262 & $N(27,8,7)$ \\
\hline Pedestrians & 1.06 & 0.356 & $N(7,2)$ \\
\hline
\end{tabular}

\section{Computational experiment}

The purpose of the computational experiment is to find the current and the maximum load level for "Vladykino" and "Kutuzovo" transport hubs, as well as 
to calculate the efficiency parameters for different intensity of the incoming passenger flows.

Example 1. Here we deal with "Vladykino" hub. In terms of queuing theory the model has form

$$
\begin{gathered}
\text { BMAP/M } / 19 / 1500 \rightarrow * / M / 19 / 9500 \rightarrow \\
\rightarrow * / G^{X} / 3 / 7500 .
\end{gathered}
$$

The characteristics of the incoming flow are presented in Table 7 , the intensity $\lambda=3$. There are three types of requests that have an individual probability of arrival. The number of channels, the queue length, average time and type of service for each phase are shown in Table 3 and Table 5.

To assess the efficiency of the transport hub operating we should find following characteristics. Let $T_{\text {sist }}$ be an average sojourn time in the system, $P_{\text {loss }}$ be a loss probability, $\bar{k}$ be an average number of occupied channels; $l$ be an average length of queue, $w$ be an average idle time in a queue; $t_{p h}$ be an average sojourn time in the phase; $t_{\text {lock }}$ be an average time of channel blocking. Table 9 shows the results of simulation.

Table 9. Characteristics of Vladykino hub for $\lambda=3$.

\begin{tabular}{|c|c|c|c|c|c|}
\hline & Arrived & Accepted & $T_{\text {sist }}$ & \multicolumn{2}{|c|}{$P_{\text {loss }}$} \\
\hline Groups & 352 & 352 & \multirow{2}{*}{262,77} & \multicolumn{2}{|c|}{0} \\
\hline Requests & 11945 & 11945 & & \multicolumn{2}{|c|}{} \\
\hline & $\bar{k}$ & $l$ & $w$ & $t_{p h}$ & $t_{\text {lock }}$ \\
\hline Phase 1 & 5,01 & 47,60 & 32,51 & 35,52 & 0 \\
\hline Phase 2 & 4,60 & 4,74 & 5,63 & 8,40 & 0 \\
\hline Phase 3 & 2,61 & 193,11 & 115,03 & 205,91 & - \\
\hline \multicolumn{2}{|c|}{$\begin{array}{c}\text { Average queue length } \\
\text { for Phase 3 }\end{array}$} & QS I & QS II & QS III \\
\cline { 2 - 5 } & 30,90 & 156,61 & 5,46 \\
\hline
\end{tabular}

Move on now to Kutuzovo hub. Here the intensity of the incoming $B M A P$-flow $\lambda=2$. Probabilities of arrival of passenger flows are shown in Table 8 . In terms of queuing theory the model has form

$$
\begin{gathered}
\text { BMAP/M } / 18 / 1500 \rightarrow * / M / 18 / 2000 \rightarrow \\
\rightarrow * / G^{X} / 3 / 5000 .
\end{gathered}
$$

Table 10 shows the results of simulation.

Table 10. Characteristics of Kutuzovo hub for $\lambda=2$.

\begin{tabular}{|c|c|c|c|c|c|}
\hline & Arrived & Accepted & $T_{\text {sist }}$ & \multicolumn{2}{|c|}{$P_{\text {loss }}$} \\
\hline Groups & 222 & 222 & \multirow{2}{*}{278,40} & \multicolumn{2}{|c|}{0} \\
\cline { 1 - 5 } Requests & 11880 & 11880 & & \multicolumn{2}{|c|}{} \\
\hline & $\bar{k}$ & $l$ & $w$ & $t_{p h}$ & $t_{\text {lock }}$ \\
\hline Phase 1 & 5,04 & 78,82 & 51,28 & 54,35 & 0 \\
\hline
\end{tabular}

Table 10 (Continued)

\begin{tabular}{|c|c|c|c|c|c|}
\hline Phase 2 & 4,69 & 7,38 & 6,32 & 9,18 & 0 \\
\hline Phase 3 & 2,85 & 191,51 & 117,03 & 214,87 & - \\
\hline \multicolumn{3}{|c|}{$\begin{array}{c}\text { Average queue length } \\
\text { for Phase 3 }\end{array}$} & QS I & QS II & QS III \\
\cline { 2 - 6 } & 27,90 & 149,18 & 14,43 \\
\hline
\end{tabular}

The simulation results show that QS III (bus station) is underloaded and has small average queue length. This is explained by the fact that we consider only passengers passing from the terminal to bus stations, whereas passengers heading straight to the buses are not counted.

In general, the characteristics of both hubs are similar, but the average queue length for phases 1 and 2 for "Kutuzovo" is approximately twice as large. The reason is the smaller number of channels in phases 1 and 2 (4 instead of 5) which serve passenger flow II.

Experiments do not take into account the transition time between phases. According to the field observation, the transition time from the metro to the MCC station is approximately 5 minutes for Vladykino hub and 3 minutes for Kutuzovo hub. The average time for buying tickets is determined separately. There are seven ticket offices, five of which are automatic. According to statistics, the average service time is two requests per minute. Then we obtain that average time for buying tickets is two minutes for considered incoming flow. Consequently, the average sojourn time in the hub is 9.5 and 7.4 minutes for Vladykino and Kutuzovo, respectively. Such time is acceptable.

Example 2. The purpose of this example is to determine the maximum permissible load of considered hubs.

As a result of the series of tests, we found that if the intensity increases 1.33 times then the average queue in QS II (phase 3) increases 4 times, and the average sojourn time in the system increases by $19 \%$. With an increase in intensity by a factor of 1.5 , the average length of the queue in phase 2 increases 12 times and in QS II (Phase 3) increases 6 times. The average sojourn time exceeds 15 minutes.

With a twofold increase in the intensity of passenger arrival $(\lambda=6)$, the average sojourn time in Vladykino hub will exceed 23 minutes (taking into account the transition time between phases) and the average length of the queues in phases 1 and 2 is more than 500 people. This means that five turnstiles at the entrance and exit to the MCC platform are not enough. Accordingly, here there is the longest queue (QS II). Note that the loss probability becomes nonzero and 
sojourn time is about 18 minutes. Table 11 shows the results of simulation.

Table 11. Characteristics of Vladykino hub for $\lambda=6$.

\begin{tabular}{|c|c|c|c|c|c|}
\hline & Arrived & Accepted & $T_{\text {sist }}$ & \multicolumn{2}{|c|}{$P_{\text {loss }}$} \\
\hline Groups & 721 & 716 & \multirow{2}{*}{1069,53} & \multicolumn{2}{|c|}{0,01} \\
\cline { 1 - 5 } Requests & 23849 & 22730 & & $t_{p h}$ & $t_{\text {lock }}$ \\
\hline & $\bar{k}$ & $l$ & $w$ & 196,30 & 0 \\
\hline Phase 1 & 8,96 & 585,70 & 193,20 & 196,3 & 241,73 \\
\hline Phase 2 & 8,59 & 549,10 & 207,06 & 210,39 & - \\
\hline Phase 3 & 2,87 & 1471,71 & 569,03 & 662,85 & \\
\cline { 1 - 5 } $\begin{array}{c}\text { Average queue length } \\
\text { for Phase 3 }\end{array}$ & QS I & QS II & QS III \\
\cline { 2 - 5 } & 54,28 & 1404,43 & 12,88 \\
\hline
\end{tabular}

Now we carry out a similar experiment for Kutuzovo hub. Assume the intensity is twofold $(\lambda=4)$. Table 12 shows the results of simulation.

Table 12. Characteristics of Kutuzovo hub for $\lambda=4$.

\begin{tabular}{|c|c|c|c|c|c|}
\hline & Arrived & Accepted & $T_{\text {sist }}$ & \multicolumn{2}{|c|}{$P_{\text {loss }}$} \\
\hline Groups & 481 & 434 & \multirow{2}{*}{1032,10} & \multicolumn{2}{|c|}{0,10} \\
\cline { 1 - 5 } Requests & 25530 & 19626 & & $t_{p h}$ & $t_{\text {lock }}$ \\
\hline & $\bar{k}$ & $l$ & $w$ & 467,43 & 0 \\
\hline Phase 1 & 7,77 & 1223,91 & 464,35 & 463 & 425,47 \\
\hline Phase 2 & 7,75 & 198,03 & 79,15 & 82,29 & - \\
\hline Phase 3 & 2,96 & 961,51 & 391,80 & 482,36 & - \\
\hline \multicolumn{3}{|c|}{$\begin{array}{c}\text { Average queue length } \\
\text { for Phase 3 }\end{array}$} & QS I & QS II & QS III \\
\cline { 2 - 5 } & 43,18 & 838,53 & 79,76 \\
\hline
\end{tabular}

An insufficient number of turnstiles at the MCC platform leads to long queue in Phase 1. This explains the high loss probability $(10 \%)$. By the average length of the queue in front of the system, we can argue that the MCC trains cannot cope with the given load level. The average sojourn time is more than 20 minutes (taking into account the transition time between phases).

Summarizing, we note that in present time both transport hubs operate without overloads. They have marginal load capacity for an average passenger flow of 11-12 thousand people at rush hours. Achieving such a passenger flow is planned by 2025 . With the increase in the number of passengers over 18 thousand people at rush hour (1.5 times increase in intensity) transition ceases to be comfortable and exceeds 15 minutes.

We found two "bottlenecks" in both hubs. The first is a small number of turnstiles at the entrance (exit) to the MCC platform, especially in Kutuzovo hub. The second is the low capacity of MCC trains. In the first case, it is necessary to increase the number of turnstiles up to 6 or temporarily invert the direction of the passengers moving for some of them. In the second case, it is necessary to increase the number of wagons (if technically possible), or to reduce the inter-train interval.

\section{Conclusions}

The article proposes a new approach to solving urgent problems of modern urban transport - scenario modeling of the work of passenger hubs. As a mathematical apparatus, we use multiphase queuing systems with an incoming $B M A P$-flow. Since it is difficult to analyze such systems analytically, we realize this approach in the form of a simulation model. The model is tested on two passenger hubs in Moscow. The results of the computational experiment show that the model makes it possible to give an overall assessment of the effectiveness of the transport hub. Also, the model allows determining the current parameters of operation, calculating the maximum permissible load and finding "bottlenecks" in the structure of passenger transport.

Further development of the presented approach is aimed at improving the functionality of the software. For example, modeling taking into account the daily fluctuations in passenger flows and transport traffic, various contingency situations, including the possibility of failure of service channels and their subsequent repair.

\section{Acknowledgments}

The work is partially supported by RFBR, projects Nos 18-07-00604, 16-06-00464. 


\section{References}

1. R. V. Vukan, Transportation for Livable Cities (Center for Urban Policy Research, New Jersey, 1999).

2. A. Monzón, S. Hernández and F. Di, Ciommo Efficient urban interchanges: the City-HUB model, Transportation Research Procedia. 14 (2016) pp. 1124-1133.

3. D. Banister, The sustainable mobility paradigm, Transport Policy. 15(2) (2008) 73-80.

4. R. Cervero and J. Murakami, Rail and Property Development in Hong Kong, Urban Studies. 46(10) (2009) 2019-2043.

5. B. Van Wee, Urban Form and Transport Accessibility, Journal of Environmental Policy \& Planning. 15(2) (2013) 323-324.

6. J. Medhi, Stochastic models in queueing (Academic press, London, 2003).

7. G. Bolch (eds.), Queueing Networks and Markov Chains: Modeling and Performance Evaluation with Computer Science Applications (John Wiley \& sons, New York, 2006).

8. R. J. Spillar, Park-and-Ride Planning and Design Guidelines (Parsons Brinckerhoff Quade \& Douglas Inc., New York, 1997).

9. D. M. Lucantoni, New results on single server queue with a batch Markovian arrival process, Communications in Statistics. Stochastic Models. 7 (1991) 1-46.

10. M. F. Neuts, A versatile markovian point process, J. of Application Problems (1979) 746-779. 